\title{
A Study of Sensitivity of a Least Mean Square Filter on its Tap Weight Vector Length and Step Size in Adaptively Cancelling Noise in an Electrocardiogram Signal
}

\author{
Sushmita Haldar \\ Department of Electronics and \\ Communication Engineering, \\ Maulana Azad National \\ Institute of Technology, Bhopal \\ Madhya Pradesh, India
}

\begin{abstract}
Electrocardiogram (ECG) is a procedure that records the activity of the heart and presents it as an electrical signal. It is an immensely important diagnostic tool since any deviation from the characteristic shape of an ECG may point to a cardiac anomaly, making it important for instrument and processing to be very accurate. Since the nature of noise that could corrupt the ECG is non-stationary, Adaptive Noise Cancellation (ANC) filters are required. In this paper, the Adaptive Noise Cancellation algorithm Least Mean Squares (LMS) has been implemented to reduce the noise in a corrupted ECG signal. The parameter sensitivity of the filter on its result had been studied, how the values of tap weight vector length and step size of the filter influence the quality of the resultant signal of the filter. This has been done through simulations in MATLAB, the noise have been simulated in MATLAB and the ECG signals have been collected from the ECG-ID database at PhysioNet. For various values of tap weight vector length and step size the performance of the LMS filter is analyzed in terms of PRD and MSE and the observations are tabulated. At the end of this study parameter values are suggested that render the most optimum results.
\end{abstract}

\section{GENERAL TERMS}

Adaptive Noise Cancellation, Parameter Sensitivity.

\section{Keywords}

ECG, ANC, LMS, tap weight vector length, step size, PRD, MSE.

\section{INTRODUCTION}

An electrocardiogram(ECG) is a procedure that measures the heart activity that is, its contractions and relaxations and displays this as an electrical signal. The signal hence produced can be used to detect cardiac abnormalities since the ECGs of healthy hearts have a characterictic shape and any deviation from that shape may be due to an irregularity in the heart function indicating a cardiac anomaly. Some heart problems that can be diagnosed through an ECG include enlargement of the heart, congenital defects, arrhythmia, coronary occlusion and abnormal positions of the heart. The importance of the ECG in medical diagnosis makes it quintessencial for the signal to be completely accurate, since any deviation from the characteristic shape due to noise could lead to misdiagnosis. This calls for the need of noise cancellation methods with extremely precise results. The noises that are majorly responsible for the distortions in the ECG are white noise, power line interface, noises due to electrode motion and muscle artifact. However these noises being non stationary in nature cannot be filtered out using constant coeficient filters like Finite Impulse Response (FIR) and Infinite Impulse Response Filters (IIR). Since these filters cannot change their coeficients according to the randomly changing nature of the noise signal, adaptive noise cancellation techniques[1]-[2] have been employed to reduce the noise from non stationary noise corrupted ECGs. Least Mean Square (LMS) is one such technique[3]-[6]. The performance of an LMS filter depends on how its parameters have been chosen. The mangitude of noise reduction by an LMS filter is sensitive to its parameters like step size and tap weight vector length[7]-[8].

This paper analyses the performance of an LMS filter as its parameters, step size and tap weight vector length are varied with the intent of an improvement in result which is represented in terms of simulations on MATLAB, MSE and PRD. Depending on the results parameters for the most optimum results are determined.

\section{THE LMS ADAPTIVE NOISE \\ CANCELLATION ALGORITHM}

The figure shows the block diagram of a basic Adaptive Noise Canceller(ANC). The signals in the figure $s(k)$ and $n(k)$ are the uncorrupted signal and the noise respectively. Another signal which is input to the ANC in n'( $\mathrm{k}$ ) is a reference signal which is uncorrelated to $\mathrm{s}(\mathrm{k})$ and correlated to the noise $\mathrm{n}(\mathrm{k})$.

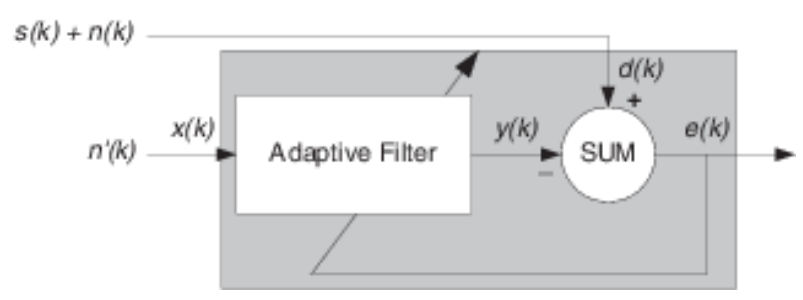

Figure 1: Block diagram of an adaptive noise canceller.

$\mathrm{d}(\mathrm{k})=\mathrm{s}(\mathrm{k})+\mathrm{n}(\mathrm{k})$
$\mathrm{e}(\mathrm{k})=\mathrm{d}(\mathrm{k})-\mathrm{y}(\mathrm{k})$

where, $y(k)$ is a signal produced by a conventional filter as the with the tap weights $w_{n}$ and $n^{\prime}(k)$ as input. The error $e(r)$ must be minimized hence $e(r)$ is fed back to determine the set of filter coeficients $\mathrm{w}_{\mathrm{n}+1}$ for the next iteration. 
$\mathrm{y}(\mathrm{n})=\sum \mathrm{w}_{\mathrm{n}} \mathrm{n}^{\prime}(\mathrm{k}-\mathrm{n})$

$\mathrm{E}(\mathrm{k})=\mathrm{s}(\mathrm{k})+\mathrm{n}(\mathrm{k})-\mathrm{y}(\mathrm{k})$

Squaring both the sides,

$\mathrm{e}^{2}(\mathrm{k})=\mathrm{s}^{2}(\mathrm{k})+(\mathrm{n}(\mathrm{k})-\mathrm{y}(\mathrm{k}))^{2}+2 \mathrm{~s}(\mathrm{k})(\mathrm{n}(\mathrm{k})-\mathrm{y}(\mathrm{k}))$

Since $y(k)$ will also be uncorrelated with $s(k)$, the previous equation can be rewritten as

$\mathrm{e}^{2}(\mathrm{k})=\mathrm{s}^{2}(\mathrm{k})+(\mathrm{n}(\mathrm{k})-\mathrm{y}(\mathrm{k}))^{2}$

In the LMS algorithm the objective is to obtain an output $\mathrm{e}(\mathrm{k})$ with the least value of the sqaure of $(n(k)-y(k))$. The lesser this value the more accurate noise cancellation.

This optimization is controlled by e(n) itself, every time it is fed back the tap weights are adjusted to minmize the $n(k)-$ $y(k)$ from the previous iterartion. The tap weights are adjusted as,

$\mathrm{w}_{\mathrm{n}+1}=\mathrm{w}_{\mathrm{n}}+\mu \mathrm{e}(\mathrm{k}) \mathrm{n}^{\prime}(\mathrm{k})$

and, $\mathrm{y}(\mathrm{k})=\sum \mathrm{w}_{\mathrm{n}} \mathrm{n}^{\prime}(\mathrm{k}-\mathrm{n})$

As the iterations are carried out the signal is gradually filtered with successive samples. The choice parameter $\mu$, the step size and the tap weight vector length impress upon the nature of the filter's result. Thus theses two parameters must be varied so as to get optimum results.

\section{EXPERIMENTAL FINDINGS}

In this section the simulations of the proposed idea have been presented. These simulations have been carried out on MATLAB. The ECGs have been collected from the ECG-ID database of PhysioNet and the White Gaussioan Noise has been simulated in MATLAB. The ECGs were 10 second long digital signals and for the experiment the first 1000 samples have been used with amplitudes in millivolts. ECG signals are corrupted using White Gaussian Noise and this corrupted signal has been filtered using the LMS filter while varying $\mu$ and the tap weight vector length to achieve that combination of these two parameters which render the minimum deviation from the original signal.

Figure 2 shows the ECG signal corrupted by White Gaussian Noise being filtered out by the four different LMS adaptive filter with tap weight vector lengths L1, L2, L3 and L4 which are $32,24,16$ and 8 respectively, keeping all others parameters uniform. It can be inferred that decreasing the tap weight vector length improves the results sufficiently.
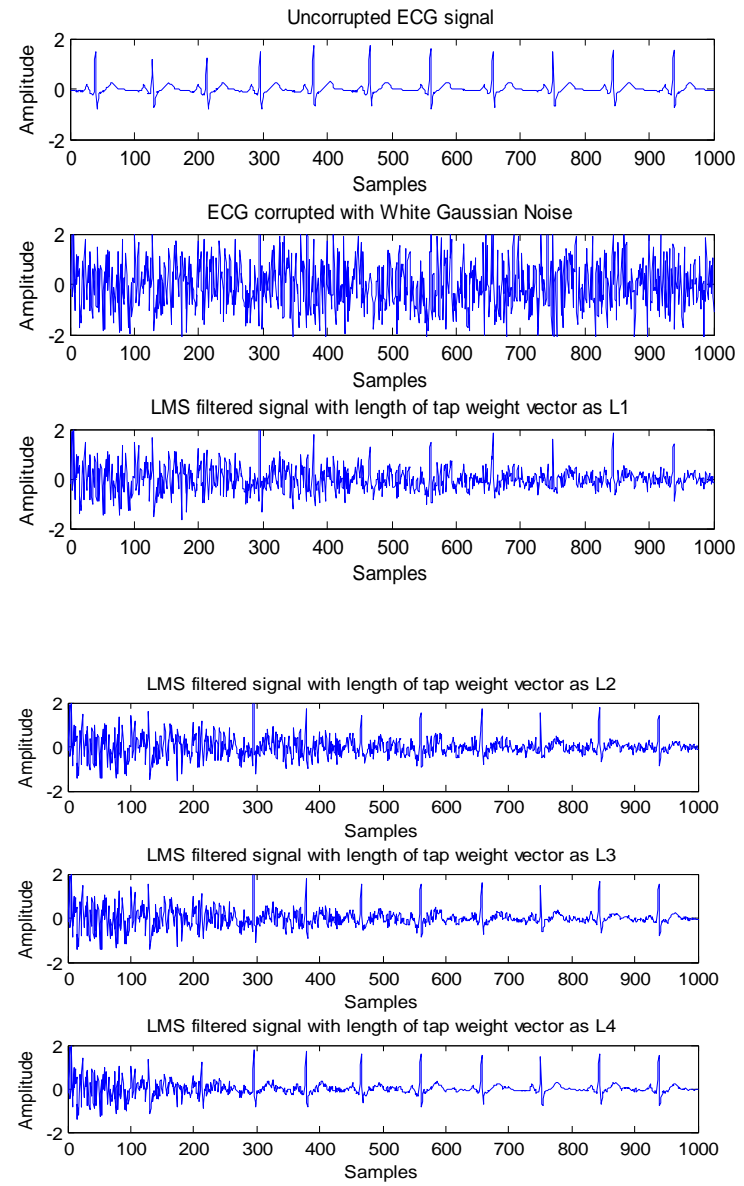

Figure 2: The uncorrupted ECG followed by ECG corrupted by White Gaussian Noise and LMS filtered using tap weight vector lengths $L 1, L 2, L 3$ and L4.

Tables 1 and 2 show the numerical analysis of performance of LMS filters of different tap weight length vectors in terms of PRD and MSE. It can be inferred from these tables that the filter works most accurately for tap weight vector length 8 .

Table 1: Analysis in terms of PRD improvement with respect to tap weight vector length

\begin{tabular}{|l|c|c|c|c|}
\hline & \multicolumn{4}{|c|}{ PRD values } \\
\hline ECG/length & L1=32 & L2=24 & L3=16 & L4=8 \\
\hline ECG 1 & 70.2 & 52.1 & 39.44 & 34 \\
\hline ECG 2 & 69.7 & 50.6 & 41.72 & 25.4 \\
\hline ECG 3 & 54.5 & 49.1 & 44.55 & 24.2 \\
\hline Average & 64.8 & 50.6 & 41.9 & 27.8 \\
\hline
\end{tabular}

Table 2: Analysis in terms of MSE improvement with respect to tap weight vector length

\begin{tabular}{|l|c|c|c|c|}
\hline & \multicolumn{4}{|c|}{ MSE values } \\
\hline ECG/length & L1=32 & L2=24 & L3=16 & L4=8 \\
\hline ECG 1 & 0.89 & 0.83 & 0.764 & 0.7 \\
\hline ECG 2 & 1.42 & 1.34 & 1.237 & 1.13 \\
\hline ECG 3 & 1.08 & 1.03 & 0.971 & 0.91 \\
\hline Average & 1.13 & 1.07 & 0.991 & 0.91 \\
\hline
\end{tabular}


Figure 3 shows the ECG signal corrupted by White Gaussian Noise being filtered out by the three different LMS adaptive filter with step sizes S1, S2 and S3 which are 0.01, 0.05 and 0.1 respectively, keeping all others parameters uniform. The tap weight vector length is 8 for all the filters.
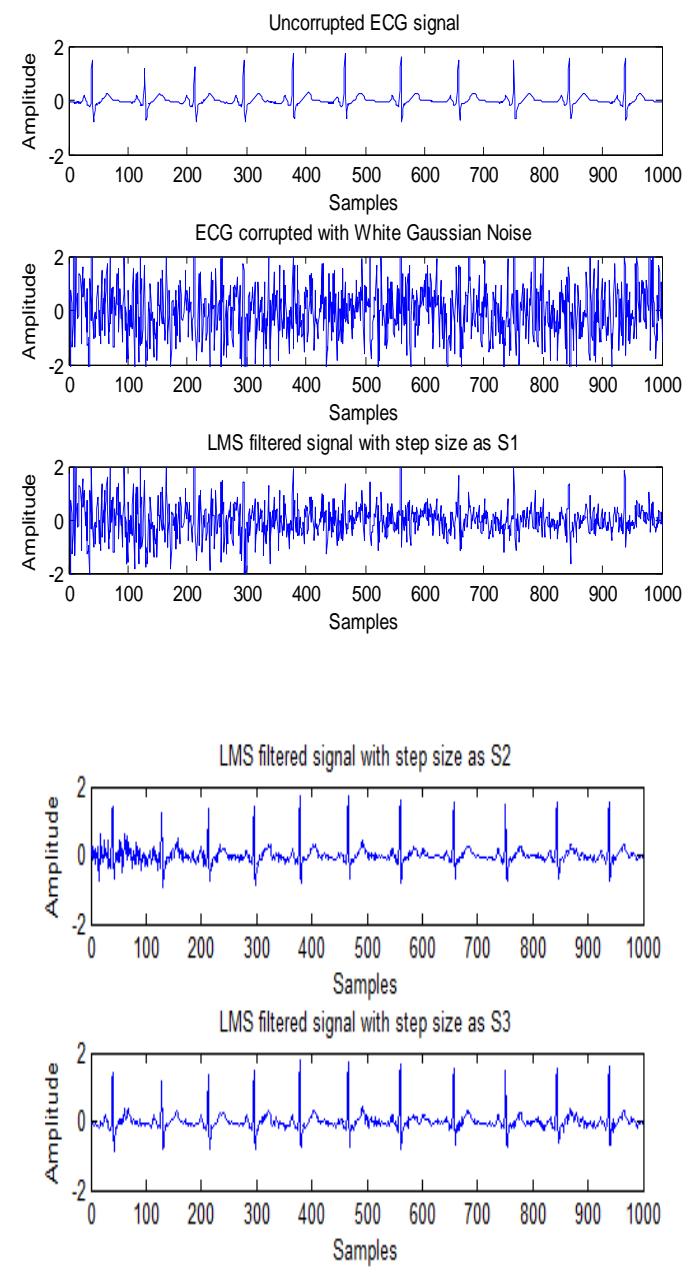

Figure 3 The uncorrupted ECG followed by ECG corrupted by White Gaussian Noise and LMS filtered using step sizes S1, S2 and S3.

Tables 3 and 4 show the numerical analysis of performance of LMS filters of different step sizes in terms of PRD and MSE. It can be inferred from these tables that the filter works most accurately for the step size 0.05 .

Table 3: Analysis in terms of PRD improvement with respect to step size

\begin{tabular}{|l|c|c|c|}
\hline & \multicolumn{3}{|c|}{ PRD values } \\
\hline ECG/ $\boldsymbol{\mu}$ & $\mathbf{0 . 0 1}$ & $\mathbf{0 . 0 5}$ & $\mathbf{0 . 1}$ \\
\hline ECG 1 & 75.4 & 19.6 & 22.79 \\
\hline ECG 2 & 61.9 & 17.6 & 21.96 \\
\hline ECG 3 & 68 & 19.3 & 25.07 \\
\hline Average & 68.5 & 18.8 & 23.27 \\
\hline
\end{tabular}

Table 4: Analysis in terms of MSE improvement with respect to step size

\begin{tabular}{|l|c|c|c|}
\hline & \multicolumn{3}{|c|}{ MSE values } \\
\hline ECG/ $\boldsymbol{\mu}$ & $\mathbf{0 . 0 1}$ & $\mathbf{0 . 0 5}$ & $\mathbf{0 . 1}$ \\
\hline ECG 1 & 0.94 & 0.63 & 0.652 \\
\hline ECG 2 & 1.36 & 1.01 & 1.051 \\
\hline ECG 3 & 1.18 & 0.84 & 0.866 \\
\hline Average & 1.16 & 0.83 & 0.857 \\
\hline
\end{tabular}

\section{CONCLUSIONS}

LMS filter shows plausible results in filtering non stationary signals. The effectiveness is sensitive to its parameters, tap weight vector length and step size. As different filters have been implemented to minimize the noise in the corrupted signal varying one parameter while keeping other constant and vice versa, a combination of step size and tap weight vector length of 0.05 and 8 respectively has shown to improve the results by a large magnitude.

\section{REFERENCES}

[1] Simon Haykin: "Adaptive Filter Theory", Third Edition, Prentice Hall, Inc., Upper Saddle River, NJ, 1996.

[2] John G. Proakis, Dimitris G. Manolakis: "Digital Signal Processing”, Fourth Edition, Pearson, pp. 905-907.

[3] T. Pitchaiah, P. V. Sridevi and S. K. Rao, "Adaptive noise cancellation using LMS algorithm in Monte Carlo simulation," Electronics and Communication Systems (ICECS), 2015 2nd International Conference on, Coimbatore, 2015, pp. 368-372.

[4] G. Makwana and L. Gupta, "De-noising of Electrocardiogram (ECG) with Adaptive Filter Using MATLAB," Communication Systems and Network Technologies (CSNT), 2015 Fifth International Conference on, Gwalior, 2015, pp. 511-514.

[5] A. C. Mugdha, F. S. Rawnaque and M. U. Ahmed, "A study of recursive least squares (RLS) adaptive filter algorithm in noise removal from ECG signals,"Informatics, Electronics \& Vision (ICIEV), 2015 International Conference on, Fukuoka, 2015, pp. 1-6.

[6] U. Biswas, A. Das, S. Debnath and I. Oishee, "ECG signal denoising by using least-mean-square and normalised-least-mean-square algorithm based adaptive filter,"Informatics, Electronics \& Vision (ICIEV), 2014 International Conference on, Dhaka, 2014, pp. 1-6.

[7] A. Mousa, M. Qados and S. Bader, "Adaptive noise cancellation algorithms sensitivity to parameters," Multimedia Computing and Systems (ICMCS), 2011 International Conference on, Ouarzazate, 2011, pp. 1-5.

[8] R. Nagal, P. Kumar and P. Bansal, "Performance analysis of least mean square algorithm for different step size parameters with different filter order and iterations,"Recent Developments in Control, Automation and Power Engineering (RDCAPE), 2015 International Conference on, Noida, 2015, pp. 326-331. 